\title{
Prevalence of extensively drug-resistant tuberculosis in a Chinese multidrug-resistant TB cohort after redefinition
}

\author{
Cong Yao ${ }^{1 \dagger}$, Haiping Guo ${ }^{1 \dagger}$, Qiang $\mathrm{Li}^{2 \dagger}$, Xuxia Zhang ${ }^{1}$, Yuanyuan Shang ${ }^{1}$, Tongxin $\mathrm{Li}^{3}$, Yufeng Wang ${ }^{4}$, \\ Zhongtan Xue ${ }^{4}$, Lu Wang ${ }^{1}$, Liang $\mathrm{Li}^{1}$ and Yu Pang ${ }^{1 *}$ (])
}

\begin{abstract}
Objectives: Recently, the definition of extensively drug-resistant TB (XDR-TB) has been revised. In this study, we conducted a descriptive and retrospective study to determine the prevalence of XDR-TB in a Chinese multidrug-resistant TB (MDR-TB) cohort.

Methods: Broth microdilution method was performed to determine in vitro susceptibilities of Mycobacterium tuberculosis (MTB) isolates to (FQs), bedaquiline (BDQ) and linezolid (LZD). The putative drug target genes conferring drug resistance were screened by DNA sequencing.

Results: A total of 425 MDR-TB isolates were included from 13 pilots in China. LZD and BDQ resistance were noted in $30(7.1 \%)$ and $10(2.4 \%)$ isolates. On the basis of latest definitions, $114(26.8 \%)$ were MDR-TB, 282 (66.4\%) were preXDR-TB, and 29 (6.8\%) were XDR-TB. Among 311 FQ-resistant isolates, 265 harbored genetic mutations within QRDRs. The most common mutations were observed at codon 94 of gyrA, accounting for $47.2 \%$ of FQ-resistant MTB isolates. Only mutations within the Rv0678 gene were found to confer BDQ resistance in our cohort, conferring $40.0 \%$ of BDQ resistance. For $L Z D$ resistance, $53.3 \%$ of LZD-resistant isolates carried genetic mutations in rp/C or $23 S$ rRNA. The most frequent mutation was Cys154Arg in the rp/C gene. In addition, we recorded two MDR-TB patients with resistance to both $B D Q$ and LZD, of which one patient experienced continuous positive culture of MTB despite inclusion of efficacious moxifloxacin.
\end{abstract}

Conclusion: Our results demonstrate that the low prevalence of XDR-TB holds great promise for MDR-TB treatment with WHO-endorsed regimens containing BDQ-LZD combination, whereas the high prevalence of FQ-resistance in MDR-TB patients warrants national attention.

Keywords: Mycobacterium tuberculosis, XDR, Susceptibility

*Correspondence: pangyupound@163.com

${ }^{\dagger}$ Cong Yao, Haiping Guo and Qiang Li have contributed equally to this work.

${ }^{1}$ Department of Bacteriology and Immunology, Beijing Chest Hospital, Capital Medical University/Beijing Tuberculosis and Thoracic Tumor Research Institute, Postal No 9, Beiguan Street, Tongzhou District, Beijing 101149, People's Republic of China

Full list of author information is available at the end of the article

\section{Introduction}

Tuberculosis, caused by Mycobacterium tuberculosis (MTB) complex, continues to be a global public health priority $[1,2]$. The World Health Organisation (WHO) estimates that approximately 10.0 million people developed TB and 1.4 million people died from this disease in 2019 [1]. The emergence of multidrug-resistant tuberculosis (MDR-TB; resistant to at least isoniazid and rifampin), with an estimated burden of $78 \%$ original author(s) and the source, provide a link to the Creative Commons licence, and indicate if changes were made. The images or other third party material in this article are included in the article's Creative Commons licence, unless indicated otherwise in a credit line to the material. If material is not included in the article's Creative Commons licence and your intended use is not permitted by statutory regulation or exceeds the permitted use, you will need to obtain permission directly from the copyright holder. To view a copy of this licence, visit http://creativecommons.org/licenses/by/4.0/. The Creative Commons Public Domain Dedication waiver (http://creativeco mmons.org/publicdomain/zero/1.0/) applies to the data made available in this article, unless otherwise stated in a credit line to the data. 
rifampicin-resistant TB cases worldwide, has jeopardised TB control and subverted the goals of the WHO's END TB Strategy $[1,3]$. Because of inherent resistance to the two most potent anti-TB drugs, MDR-TB treatment requires the use of second-line drugs that are less effective, more toxic, and costlier than first-line regimens [4, 5]; however, the overall rate of treatment success among MDR-TB patients is currently $57 \%$ [1]. This unsatisfactory outcome is expected to worsen the epidemic of this severe form of TB. Therefore more efforts are urgently needed for new and effective drugs to improve the chemotherapy of MDR-TB [6].

Over the past decades, several new or re-purposed agents antimicrobial hold promise for MDR-TB treatment, such as bedaquiline (BDQ), linezolid (LZD) and delamanid [6-9]. Recent clinical trials have demonstrated that higher treatment success rates are achievable by inclusion of these new or re-purposed agents in the regimens [10]. Similarly, A study in South Africa demonstrates that bedaquiline (BDQ) is a safe drug and is associated with the high success rate for the MDR-TB and XDR-TB cohort even in high HIV burden areas [11, 12]. Recently, the WHO released the updated guidelines on the MDR-TB treatment on the basis of new experimental and observational evidence, in which the late-generation fluoroquinolones (FQs, i.e., levofloxacin and moxifloxacin), linezolid and bedaquiline are classified as preferred Group A drugs [13]. Subsequently, the definition of extensively drug-resistant TB (XDR-TB) has been revised by the WHO Global TB Programme, aiming to enable access to more effective treatment options for patients afflicted with drug-resistant strains [14]. The updated XDR-TB is defined as infection with an MDR-TB strain that is also resistant to any fluoroquinolone and at least one additional Group A drug. This redefinition points to increasing progression of the severity of the disease, and predicts poor clinical outcomes. The appropriate drug susceptibility testing (DST) methods are required to optimize the use of Group A drugs to improve the treatment of MDR-TB. Unfortunately, the commercial DST methods remain limited to assess in vitro susceptibility of MTB isolates to bedaquiline and linezolid. The national surveillance of anti-TB resistance in MDR-TB is thus essential to assist countries in planning the scale-up of patient management.

Despite a significant achievement in tackling the TB epidemic over the past years, China has a serious MDRTB burden, with an estimated 74\% rifampicin-resistant/ MDR-TB cases in 2019 [1, 15]. Findings on temporal surveillance demonstrated that the prevalence of MDR-TB has dramatically increased in China [16]. The spread of MDR- and XDR-TB has been a major threat from both a clinical and a public health perspective. Although recent changes in the WHO recommendations for treatment of RR/MDR-TB patients have replaced injectables with bedaquiline in preferred therapy [17], the treatment of these patients majorly relies on long-course regimens stipulated by the WHO due to limited accessibility to bedaquiline. Only $54 \%$ of RR/MDR-TB cases who started treatment achieved a favorable outcome, which was recognized as a threat to TB control efforts in this country. To date, we still lack national data regarding the prevalence of XDR-TB in China. To address this concern, we conducted a study to determine in vitro susceptibilities of MTB isolates to FQs, bedaquiline and linezolid in a Chinese MDR-TB cohort. The putative drug target genes conferring drug resistance were screened by DNA sequencing.

\section{Materials and methods Bacterial isolates}

Between February 2018 and June 2019, a retrospective cohort study was conducted by inclusion of MDR-TB patients in 13 hospitals, aiming to determine clinical efficacy of MDR-TB patients treated with BDQ-containing regimens [18]. The baseline MTB isolates were used for in vitro drug susceptibility testing. The positive cultures were stored in $7 \mathrm{H} 9$ medium supplemented with $10 \%$ of oleic acid-albumin-dextrose-catalase (OADC) and 5\% glycerol. prior to determining minimum inhibitory concentration (MIC) values, the isolates were recovered on Löwenstein-Jensen $(\mathrm{L}-\mathrm{J})$ medium for 4 weeks at $37^{\circ} \mathrm{C}$.

\section{Minimum inhibitory concentration}

The MICs of MTB isolates to antimicrobial agents were assessed with Middlebrook $7 \mathrm{H} 9$ broth microdilution using the Thermo Fisher frozen microtiter plates as previously reported [19]. Briefly, the 4-week-old fresh colonies were scraped from the surface of L-J slants. Followed by vigorously votexing for $1 \mathrm{~min}$, a suspension of MTB isolate was adjusted to the turbidity of a 1.0 McFarland standard. The suspension was diluted to $1: 20$ as inoculum. Then $100 \mu \mathrm{L}$ of this inoculum was automatically added into each well of the 96-well drugcontaining plate. Plates were sealed and incubated at $37^{\circ} \mathrm{C}$ incubator for 10 days, 14 days or 21 days depending on visible growth in control well. The Sensititre Vizion sytem (Thermo Fisher Scientific, West Sussex, UK) was used to monitor the growth of mycobacteria in each well, and the MICs were automatically interpretated with SWIN ${ }^{\circledR}$ software (Thermo Fisher Scientific, West Sussex, UK). The concentrations of anti-TB drugs tested were as follows: ofloxacin (OFX, 0.12-8 $\mu \mathrm{g} /$ $\mathrm{mL}$ ), levofloxacin (LFX, 0.12-4 $\mu \mathrm{g} / \mathrm{mL}$ ), moxifloxacin (MFX, 0.06-4 $\mu \mathrm{g} / \mathrm{mL}$ ), bedaquiline $(0.008-4 \mu \mathrm{g} / \mathrm{mL}$ ), and linezolid $(0.12-8 \mu \mathrm{g} / \mathrm{mL})$. The MIC was defined 
as the lowest drug concentration inhibiting the visual growth of mycobacteria. MTB H37Rv (ATCC 27,249) was tested in each batch as a quality control strain. The MIC breakpoint concentrations were defined as $2 \mu \mathrm{g} /$ $\mathrm{mL}$ for OFX and LFX, $0.5 \mu \mathrm{g} / \mathrm{ml}$ for MFX, $0.25 \mu \mathrm{g} / \mathrm{ml}$ for $\mathrm{BDQ}$, and $1.0 \mu \mathrm{g} / \mathrm{ml}$ for LZD, respectively.

\section{DNA extraction and sequencing}

Genomic DNA of fresh MTB colonies was extracted with MasterPure ${ }^{\mathrm{TM}}$ Complete DNA\&RNA Purification kit (Lucigen corporation, Wisconsin, USA) according to the manufacturer's instruction. The partial fragments of genes conferring drug resistance were amplified for DNA sequencing, including $g y r A$ and $\operatorname{gyr} B$ for FQs, atpE, Rv0678, Rv1979c and pepQ for BDQ, and $23 S$ $r R N A$ and $r p l C$ for LZD, respectively. The genomic DNA was used as template to perform PCR reaction as follows: $25 \mu \mathrm{L}$ of 2xPCR mixture (CWBiotech, Beijing, China), $0.2 \mu \mathrm{M}$ of each primer and $1 \mu \mathrm{L}$ of template DNA. PCR program was done as previously reported [19-21]. The primer pairs used herein are listed in Additional file 1: Table S1. PCR products were sent to the RuiBiotech Company for DNA sequencing service (RuiBiotech, Beijing, China). The sequences were aligned with the corresponding genes of the reference MTB strain H37Rv using multiple sequence alignments at the National Center for Biotechnology Information (NCBI) website.

\section{Definitions}

MDR-TB was defined as patients infected with MTB strain resistant to at least rifampicin and isoniazid. PreXDR was defined as patients infected with MDR-TB plus additional resistance to any fluroquinolone. XDRTB was defined as patients infected with MDR-TB plus resistance to both FQ and at least one additional Group A drug [14].

\section{Results}

Drug susceptibility profiles of MDR-TB isolates

A total of 425 MDR-TB isolates were included in our analysis. Table 1 shows the drug susceptibility profiles of these isolates, of which, $311(73.2 \%)$ were resistant to FQ and $171(40.2 \%)$ to any second-line injectable drug (SLID). In addition, 143 (33.6\%) had multiresistance to both FQ and SLID. We further evaluated in vitro susceptibility of these isolates with broth microdilution method. LZD and BDQ resistance were noted in $30(7.1 \%)$ and $10(2.4 \%)$ isolates. On the basis of latest definitions, 114 (26.8\%) were MDR-TB, 282 (66.4\%) were pre-XDR-TB, and $29(6.8 \%)$ were XDR-TB. Among 29 XDR-TB isolates,
$25(86.2 \%)$ were resistant to LZD, $3(10.4 \%)$ to $\mathrm{BDQ}$, and 1 (3.4\%) to both LZD and BDQ (Fig. 1). Of note, resistance to LZD and BDQ was also recorded in MDR-TB group, consisting of 4 LZD-resistant and 6 BDQ-resistant isolates, respectively.

\section{Mutations in the quinolone resistance-determining regions (QRDRs) of gyrA and gyrB genes}

Mutations conferring FQ resistance were investigated by Sanger sequencing. As summarized in Table 2, among 311 FQ-resistant isolates, 265 (85.3\%) harbored genetic mutations within QRDRs. The most common mutations were observed at codon 94 of $g y r A$, where the wild-type serine was replaced with six different amino acid: Asp94Ala $(n=17)$, Asp94Asn $(n=19)$, Asp94Gly $(n=96)$, Asp94His $(n=4)$, Asp94Tyr $(n=3)$ and Asp94Val $(n=8)$, accounting for $47.3 \%(n=147)$ of FQ-resistant MTB isolates. The second most prevalent mutation was observed in codon 90, resulting in the amnio acids substitution of Ala to Val $(n=76$, $24.4 \%)$. For the gyrB gene, synonymous mutations were found in 7 (2.3\%) FQ-resistant isolates. The correlation between genetic mutations and MICs was further analyzed. Interestingly, the substitutions of Ala90Val and Ser91Pro within gyrA were associated with low-level FQ-resistance, and $27.6 \%(n=21)$ and $36.4 \%(n=12)$ of isolates tested were susceptible to MFX, respectively. In contrast, the isolates with the mutations at codon 94 had higher MIC values, which were all resistant to MFX.

\section{Mutations conferring BDQ and LZD resistance}

We analysed the mutation within genes conferring BDQ resistance. Only mutations within the $R v 0678$ gene were found to confer BDQ resistance in our cohort. Of 10 BDQ-resistant isolates, 4 (40.0\%) had Rv0678 mutations, while no mutations were noted in the other 6 isolates (Table 3). Notably, all BDQ-resistant isolates had

Table 1 Drug susceptibility profiles of MDR-TB isolates enrolled in this study

\begin{tabular}{llc}
\hline Drug susceptibility profile & No. of isolates & Proportion (\%) \\
\hline INH+RIF & 86 & 20.2 \\
INH + RIF + FQ & 168 & 39.5 \\
INH + RIF + SLID & 28 & 6.6 \\
INH + RIF + FQ + SLID & 143 & 33.6 \\
Total & 425 & 100.0 \\
\hline
\end{tabular}

$I N H$, isoniazid; RIF, rifampicin; FQ, fluoroquinolone; SLID, second-line injectable drugs 


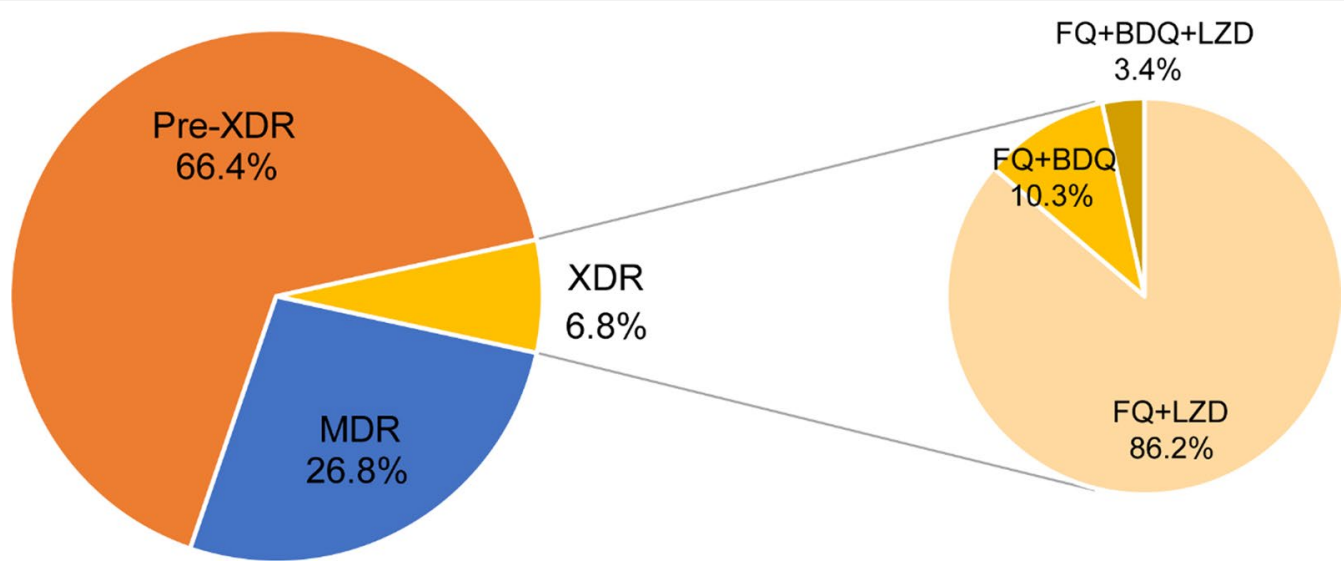

Fig. 1 Distribution of MDR-TB isolates stratified to in vitro susceptibility testing. MDR, multidrug resistance; pre-XDR, pre-extensive drug-resistance; $X D R$, extensive drug-resistance; FQ, fluroquinolone; LZD, linezolid; BDQ, bedaquiline

MICs bordering the susceptible breakpoint, varying from 0.25 to $0.5 \mu \mathrm{g} / \mathrm{ml}$.

For LZD resistance, $53.3 \%$ of LZD-resistant isolates carried genetic mutations in $\mathrm{rplC}$ or $23 \mathrm{~S}$ rRNA. The most frequent mutation was Cys154Arg $(n=12)$ in the $\mathrm{rplC}$ gene, which revealed MICs ranging from 4 to $>8 \mu \mathrm{g} / \mathrm{ml}$. In addition, two types of nucleotide substitutions in 23S rRNA were identified among 4 LZDresistant isolates, including 3 isolates with G2270T and 1 isolate with G2270C. The MICs of isolates carrying these mutations were from 4 to $8 \mu \mathrm{g} / \mathrm{ml}$ (Table 4 ).

\section{Clinical outcomes of MDR-TB with resistance to both BDQ and LZD}

In our cohort, we recorded two MDR-TB patients with resistance to both BDQ and LZD. We thus assessed the clinical outcomes of these MDR-TB patients by the administration of BDQ-containing regimens. As shown in Table 5, out of these patients, only one patient achieved successful outcome; whereas the other patient experienced continuous positive culture of MTB despite inclusion of efficacious MFX.

\section{Discussion}

Drug resistance surveillance is of importance to identify and predict the impact of new empirical anti-TB drug prescribing [22]. To our best knowledge, this was the first snapshot of the prevalence of XDR-TB among MDRTB patients in a high-burden setting. Our data demonstrate that the rate of XDR-TB was noted in $6.8 \%$ of patients afflicted with MDR-TB, which was significantly decreased from $33.6 \%$ on the basis of previous definition. This low rate of XDR-TB was majorly attributed to relatively late introduction of $\mathrm{BDQ}$ and LZD for clinical management of MDR-TB in China, thereby preventing accumulation of drug resistant mutations [18]. Consequently, this holds great promise for MDR-TB treatment with WHO-endorsed regimens containing BDQ-LZD combination.

Although the overall prevalence of XDR-TB was low, the high prevalence of FQ-resistance in MDR-TB patients warrants national attention. In a recent population-based study in China, the prevalence of MFX resistance was markedly increased from $3.0 \%$ in 2000 to $7.7 \%$ in 2010 [23]. Similar results were observed by surveillance data in Beijing, which demonstrated that a statistically significant increase in LFX resistance over the past decade [20]. This phenomenon is probably the result of overuse of FQs in the treatment of undiagnosed bacterial infections in China in view of their promising efficacy and low occurrence of adverse events. In addition, widespread use of FQs in animal and food industries has resulted in reported excessive accumulation of antibiotics in environmental water samples in China [24]. The unexpected exposure to environmental FQs is another possible explanation for increasing FQ resistance in our MDRTB cohort. Thus our primary results directly address the concern that essential interventions are required to reduce the misuse of antibiotics in clinical practice, as well as in livestock and food industries.

The acquisition of drug resistance is associated with the presence of genetic mutations conferring resistance [25]. The most frequently observed mutations in BDQ-resistant isolates were reported in $R v 0678$, a transcriptional repressor of efflux genes participating in the regulation of expression of MmpS5-MmpL5 [25, 26]. Consistent with previous investigation, mutations in $R v 0678$ were the major mechanism for BDQ resistance in our study. The high prevalence of $R v 0678$ mutation may be related to the 
Table 2 Mutations in the quinolone resistance-determining region (QRDR) of gyrA and gyrB and fluoroquinolone MICs

\begin{tabular}{|c|c|c|c|c|c|c|c|c|c|}
\hline \multirow[t]{2}{*}{ Mutations in QRDR } & \multirow[t]{2}{*}{ N (\%) } & \multirow[t]{2}{*}{$\mathrm{FQ}$} & \multicolumn{7}{|c|}{ No. of isolates with corresponding MIC (mg/L) } \\
\hline & & & 0.12 & 0.25 & 0.5 & 1 & 2 & 4 & 8 \\
\hline \multirow[t]{3}{*}{ GyrA Asp89Asn } & $2(0.6)$ & MFX & 0 & 1 & 1 & 0 & 0 & 0 & 0 \\
\hline & & OFX & 0 & 0 & 0 & 0 & 0 & 2 & 0 \\
\hline & & LFX & 0 & 0 & 0 & 0 & 1 & 1 & 0 \\
\hline \multirow[t]{3}{*}{ GyrA Ala90Val } & $76(24.4)$ & MFX & 18 & 3 & 16 & 23 & 8 & 8 & 0 \\
\hline & & OFX & 0 & 0 & 0 & 0 & 17 & 38 & 21 \\
\hline & & LFX & 0 & 0 & 0 & 0 & 56 & 20 & 0 \\
\hline \multirow[t]{3}{*}{ GyrA Ser91Pro } & 33 (10.6) & MFX & 3 & 9 & 10 & 4 & 7 & 0 & 0 \\
\hline & & OFX & 0 & 0 & 0 & 3 & 7 & 19 & 4 \\
\hline & & LFX & 0 & 0 & 0 & 4 & 25 & 4 & 0 \\
\hline \multirow[t]{3}{*}{ GyrA Asp94Ala } & $17(5.5)$ & MFX & 0 & 0 & 10 & 4 & 3 & 0 & 0 \\
\hline & & OFX & 0 & 0 & 0 & 0 & 2 & 12 & 3 \\
\hline & & LFX & 0 & 0 & 0 & 0 & 15 & 2 & 0 \\
\hline \multirow[t]{3}{*}{ GyrA Asp94Asn } & $19(6.1)$ & MFX & 0 & 0 & 4 & 4 & 7 & 4 & 0 \\
\hline & & OFX & 0 & 0 & 0 & 0 & 3 & 4 & 12 \\
\hline & & LFX & 0 & 0 & 0 & 0 & 4 & 15 & 0 \\
\hline \multirow{3}{*}{ GyrA Asp94Gly } & 96 (30.9) & MFX & 0 & 0 & 10 & 14 & 44 & 28 & 0 \\
\hline & & OFX & 0 & 0 & 0 & 0 & 3 & 9 & 84 \\
\hline & & LFX & 0 & 0 & 0 & 0 & 11 & 85 & 0 \\
\hline \multirow[t]{3}{*}{ GyrA Asp94His } & $4(1.3)$ & MFX & 0 & 0 & 0 & 0 & 0 & 4 & 0 \\
\hline & & OFX & 0 & 0 & 0 & 0 & 0 & 0 & 4 \\
\hline & & LFX & 0 & 0 & 0 & 0 & 0 & 4 & 0 \\
\hline \multirow[t]{3}{*}{ GyrA Asp94Tyr } & $3(1.0)$ & MFX & 0 & 0 & 0 & 0 & 3 & 0 & 0 \\
\hline & & OFX & 0 & 0 & 0 & 0 & 0 & 0 & 3 \\
\hline & & LFX & 0 & 0 & 0 & 0 & 0 & 3 & 0 \\
\hline \multirow{3}{*}{ GyrA Asp94Val } & $8(2.6)$ & MFX & 0 & 0 & 0 & 2 & 4 & 2 & 0 \\
\hline & & OFX & 0 & 0 & 0 & 0 & 2 & 4 & 2 \\
\hline & & LFX & 0 & 0 & 0 & 0 & 4 & 4 & 0 \\
\hline \multirow[t]{3}{*}{ GyrB Ser447Phe } & $2(0.6)$ & MFX & 2 & 0 & 0 & 0 & 0 & 0 & 0 \\
\hline & & OFX & 0 & 0 & 0 & 0 & 0 & 2 & 0 \\
\hline & & LFX & 0 & 0 & 0 & 0 & 2 & 0 & 0 \\
\hline \multirow[t]{3}{*}{ GyrB Asp461Asn } & $1(0.3)$ & MFX & 0 & 0 & 0 & 0 & 1 & 0 & 0 \\
\hline & & OFX & 0 & 0 & 0 & 0 & 0 & 0 & 1 \\
\hline & & LFX & 0 & 0 & 0 & 0 & 0 & 1 & 0 \\
\hline \multirow[t]{3}{*}{ GyrB Asp500Asn } & $1(0.3)$ & MFX & 0 & 0 & 1 & 0 & 0 & 0 & 0 \\
\hline & & OFX & 0 & 0 & 0 & 0 & 0 & 1 & 0 \\
\hline & & LFX & 0 & 0 & 0 & 0 & 1 & 0 & 0 \\
\hline \multirow[t]{3}{*}{ GyrB Ala504Thr } & $3(1.0)$ & MFX & 0 & 0 & 0 & 0 & 0 & 3 & 0 \\
\hline & & OFX & 0 & 0 & 0 & 0 & 0 & 0 & 3 \\
\hline & & LFX & 0 & 0 & 0 & 0 & 0 & 3 & 0 \\
\hline \multirow[t]{3}{*}{ WT } & $46(14.7)$ & MFX & 0 & 7 & 15 & 12 & 8 & 4 & 0 \\
\hline & & OFX & 0 & 0 & 0 & 1 & 15 & 21 & 9 \\
\hline & & LFX & 0 & 0 & 1 & 3 & 24 & 16 & 2 \\
\hline \multirow[t]{3}{*}{ Total } & $311(100.0)$ & MFX & 23 & 20 & 67 & 63 & 85 & 53 & 0 \\
\hline & & OFX & 0 & 0 & 0 & 4 & 49 & 112 & 146 \\
\hline & & LFX & 0 & 0 & 1 & 7 & 143 & 158 & 2 \\
\hline
\end{tabular}


Table 3 Profiling of genetic mutations and MICs for BDQresistant MTB isolates

\begin{tabular}{|c|c|c|c|c|c|}
\hline \multirow[t]{2}{*}{ Locus } & \multirow[t]{2}{*}{ Mutation } & \multicolumn{3}{|c|}{$\begin{array}{l}\text { No. of isolates with } \\
\text { corresponding MIC }(\mu \mathrm{g} / \\
\mathrm{ml})\end{array}$} & \multirow[t]{2}{*}{ Total (\%) } \\
\hline & & 0.25 & 0.5 & 1 & \\
\hline \multirow[t]{4}{*}{ Rv0678 } & Ala86Val & & 1 & & $1(10.0)$ \\
\hline & Ile108Thr & & 1 & & $1(10.0)$ \\
\hline & Arg109Pro & 1 & & & $1(10.0)$ \\
\hline & $\begin{array}{l}\text { Deletion A in } \\
\text { codon } 110\end{array}$ & & 1 & & $1(10.0)$ \\
\hline Wild-type & & 4 & 1 & 1 & $6(60.0)$ \\
\hline Total & & 5 & 4 & 1 & $10(100.0)$ \\
\hline
\end{tabular}

MIC, minimum inhibitory concentration; BDQ, bedaquiline; MTB, Mycobacterium tuberculosis

prior exposure to clofazimine among MDR-TB patients considering the cross-resistance between these two drugs. Of note, mutations within the $R \nu 0678$ are highly diverse, with 4 unique mutations at 4 different positions. Although the basis for the highly diverse Rv0678 mutations remains unclear, our results reveal that the DNA sequencing is more suitable for identify mutations within Rv0678 locus rather than conventional PCR-based assays, such as real-time PCR and line probe assay. We also found that approximate half of $\mathrm{BDQ}$-resistant isolates harbored no mutations within other known BDQ resistance genes. Similar results were noted in MTB isolates with LZD resistance. In addition to target gene mutations, multiple mechanisms confer drug resistance, such as cell wall permeability and efflux [27]. An experimental study by Velayati and co-workers found that the XDRTB MTB isolates had thicker cell wall than MDR and susceptible isolates though viewing the ultrastructure of cell wall [28]. Thus we hypothesize that the cell permeability-associated mechanism may play an important role of in these MTB isolates. The poor correlation between genetic mutations and phenotypic resistance forebodes unsatisfactory performance using molecular panel to diagnose resistance to these novel drugs. Further analysis is required to elucidate novel mechanisms conferring BDQ and LZD resistance, thereby boosting the development of rapid molecular diagnostics for drug resistance.

Despite limited data, we observed that a patient with resistance to both LZD and BDQ but susceptible to MFX experienced treatment failure. In a recent BDQ clinical trial, the administration of BDQ-containing regimens provides particular benefit for pre-XDR- and XDR-TB patients [18]. On one hand, the existing evidence from studies confirmed BDQ as a cornerstone in the treatment of MDR-TB patients. Although the revised definitions of XDR-TB have emphasized the importance of $\mathrm{BDQ}$, it excludes the patients at high risk of treatment failure from pre-XDR and XDR-TB group. More clinical data is required to subtly stratify drugresistant $\mathrm{TB}$ patients according to their risk of unfavorable outcomes. On the other hand, there is an urgent

Table 4 Profiling of genetic mutations and MICs for LZD-resistant MTB isolates

\begin{tabular}{|c|c|c|c|c|c|c|}
\hline \multirow[t]{2}{*}{ Locus } & \multirow[t]{2}{*}{ Mutation } & \multicolumn{4}{|c|}{ No. of isolates with corresponding MIC $(\mu \mathrm{g} / \mathrm{ml})$} & \multirow[t]{2}{*}{ Total (\%) } \\
\hline & & 2 & 4 & 8 & $>8$ & \\
\hline$r p / C$ & Cys154Arg & & 5 & 5 & 2 & $12(40.0)$ \\
\hline \multirow[t]{2}{*}{$23 S$ rRNA } & G2270C & & 1 & & & $1(3.3)$ \\
\hline & G2270T & & 2 & 1 & & $3(10.0)$ \\
\hline Wild-type & & 7 & 6 & 1 & & $14(46.7)$ \\
\hline Total & & 7 & 14 & 7 & 2 & $30(100.0)$ \\
\hline
\end{tabular}

MIC, minimum inhibitory concentration; LZD, linezolid; MTB, Mycobacterium tuberculosis

Table 5 Clinical outcomes of patients infected with resistance to both BDQ and LZD

\begin{tabular}{|c|c|c|c|c|c|c|c|}
\hline Patient ID & Patient category & $\begin{array}{l}\text { Drug } \\
\text { susceptibility }\end{array}$ & $\begin{array}{l}\text { Mutation } \\
\text { conferring BDQ } \\
\text { resistance }\end{array}$ & $\begin{array}{l}\text { Mutation } \\
\text { conferring LZD } \\
\text { resistance }\end{array}$ & Treatment regimen & $\begin{array}{l}\text { Sputum } \\
\text { culture } \\
\text { conversion }\end{array}$ & Clinical outcome \\
\hline 040,012 & Previously treated & MDR & WT & Cys154Arg (rp/C) & $\begin{array}{l}\text { Bdq/Lzd/Mfx/Cs/Am/ } \\
\text { Pto }\end{array}$ & 8 weeks & Cured \\
\hline 010,121 & Previously treated & XDR & Ile108Thr (Rv0678) & WT & $\begin{array}{l}\mathrm{Bdq} / \mathrm{Lzd} / \mathrm{Mfx} / \mathrm{Cs} / \mathrm{Cfz} / \\
\mathrm{Am}\end{array}$ & NA & Treatment failure \\
\hline
\end{tabular}


need for commercial laboratory tests of the sensitivity of tubercle bacilli to BDQ and LZD, such as phenotypic DST using MGIT, which is essential to guide the choice of chemotherapy for MDR-TB patients.

We also acknowledged several obvious limitations to our study. First, despite the enrollment of MDR$\mathrm{TB}$ isolates in our cohort, the relatively small number of MTB isolates tested may result in potential sampling bias. Second, the patients were non-responsive MDR-TB, possibly leading to overestimation of XDRTB in Chinese population. Third, the MIC values were not assessed in triplicate. Hence, the lack of repeated experiments may introduce the systematic error by microdilution method, especially for isolates with MICs close to the breakpoint. Finally, whole genome sequencing (WGS) was not conducted to identify the molecular mechanism conferring BDQ and LZD resistance among MTB isolates without known mutations. Specially, a proportion of drug-resistant isolates may be caused by the presence of heteroresistant subpopulation, which were missed by Sanger Sequencing. Further WGS analysis is of importance to elucidate the molecular mechanism underlying resistance to these agents.

In conclusion, our results demonstrate that initial XDR-TB occurs in $6.8 \%$ of MDR-TB patients in our Chinese population, whereas the high prevalence of FQ-resistance in MDR-TB patients warrants national attention. Approximately half of BDQ-resistant and LZD-resistant isolates harbor no mutations within known resistance genes. The low prevalence of XDRTB holds great promise for MDR-TB treatment with WHO-endorsed regimens containing BDQ-LZD combination.

\section{Abbreviations}

MTB: Mycobacterium tuberculosis; XDR-TB: Extensively drug-resistant TB; MDRTB: Multidrug-resistant TB; FQs: Fluoroquinolones; BDQ: Bedaquiline; INH: Isoniazid; RIF: Rifampicin; SLID: Second-line injectable drugs; MIC: Minimum inhibitory concentration; WT: Wild-type; MFX: Moxifloxacin; OFX: Ofloxacin; LFX: Levofloxacin; LZD: Linezolid; Cfz: Clofazimine; QRDRs: Quinolone resistance determining regions.

\section{Supplementary Information}

The online version contains supplementary material available at https://doi. org/10.1186/s13756-021-00995-8.

Additional file 1. Primers used in this study for PCR amplification and sequencing.

\section{Acknowledgements}

The authors acknowledge the staff at Beijing Chest Hospital for their cooperation and support.

\section{Authors' contributions}

YP: Conceptualization, Methodology, Formal analysis, Investigation, Writingoriginal draft preparation, Writing —-review and editing; LL: Conceptualization,
Methodology, Formal analysis, Investigation, Writing — review and editing; CY: Data analysis, Writing —original draft preparation. HG: Performed all experiments, Data analysis. QL: Conceptualization, Designed the project, Data analysis. XZ: Performed experiments. YS: Performed experiments. YW: Performed experiments. ZX: Performed all experiments. LW: Performed experiments. TL: Performed experiments. All authors read and approved the final manuscript.

\section{Funding}

This study was supported by the Beijing Hospitals Authority Ascent Plan (DFL20191601), the Beijing Hospitals Authority Clinical Medicine Development of Special Funding (ZYLX202122), the Capital's Funds for Health Improvement and Research (2020-1-1041), the Scientific Research Project of Beijing Educational Committee (KM202010025001) and the Chongqing Technology Innovation and Application Development Special Project (cstc2019jscx-msxmX0152), and the Medical Research Project of Chongqing Science and Technology Commission and Health Commission (2019MSXM065).

\section{Availability of data and materials}

The datasets in the present study are accessible from the corresponding author on reasonable request.

\section{Declarations}

\section{Ethics statement}

The study was approved by the Ethics Committee of the Beijing Chest Hospital, Capital Medical University. All patients provided written informed consent before they were included in this study.

\section{Competing interests}

All authors: no conflicts.

\section{Author details}

${ }^{1}$ Department of Bacteriology and Immunology, Beijing Chest Hospital, Capital Medical University/Beijing Tuberculosis and Thoracic Tumor Research Institute, Postal No 9, Beiguan Street, Tongzhou District, Beijing 101149, People's Republic of China. ${ }^{2}$ Department of Tuberculosis, Beijing Chest Hospital, Capital Medical University/Beijing Tuberculosis and Thoracic Tumor Research Institute, Beijing 101149, People's Republic of China. ${ }^{3}$ Central Laboratory, Chongqing Public Health Medical Center, Southwest University Public Health Hospital, Chongqing 400036, People's Republic of China. ${ }^{4}$ Department of Laboratory Quality Control, Innovation Alliance On Tuberculosis Diagnosis and Treatment (Beijing), Beijing 101149, People's Republic of China.

Received: 4 May 2021 Accepted: 15 August 2021

Published online: 26 August 2021

\section{References}

1. World Health Organization. Global tuberculosis report 2020. Geneva: WHO; 2020.

2. Matteelli A, Rendon A, Tiberi S, Al-Abri S, Voniatis C, Carvalho ACC, et al. Tuberculosis elimination: where are we now? Eur Respir Rev. 2018;27(148):180035.

3. Furin J, Brigden G, Lessem E, Rich M, Vaughan L, Lynch S. Global progress and challenges in implementing new medications for treating multidrugresistant tuberculosis. Emerg Infect Dis. 2016;22(3):e151430.

4. Xu C, Pang Y, Li R, Ruan Y, Wang L, Chen M, et al. Clinical outcome of multidrug-resistant tuberculosis patients receiving standardized secondline treatment regimen in China. J Infect. 2018;76(4):348-53.

5. Daley CL, Caminero JA. Management of multidrug-resistant tuberculosis. Semin Respir Crit Care Med. 2018;39(3):310-24.

6. Tiberi S, du Plessis N, Walzl G, Vjecha MJ, Rao M, Ntoumi F, et al. Tuberculosis: progress and advances in development of new drugs, treatment regimens, and host-directed therapies. Lancet Infect Dis. 2018;18(7):e183-98.

7. Diacon AH, Pym A, Grobusch MP, de los Rios JM, Gotuzzo E, Vasilyeva I, et al. Multidrug-resistant tuberculosis and culture conversion with bedaquiline. N Engl J Med. 2014;371(8):723-32. 
8. Gler MT, Skripconoka V, Sanchez-Garavito E, Xiao H, Cabrera-Rivero JL, Vargas-Vasquez DE, et al. Delamanid for multidrug-resistant pulmonary tuberculosis. N Engl J Med. 2012;366(23):2151-60.

9. Schecter GF, Scott C, True L, Raftery A, Flood J, Mase S. Linezolid in the treatment of multidrug-resistant tuberculosis. Clin Infect Dis. 2010;50(1):49-55.

10. Conradie F, Diacon AH, Ngubane N, Howell P, Everitt D, Crook AM, et al. Treatment of highly drug-resistant pulmonary tuberculosis. N Engl J Med. 2020;382(10):893-902

11. Ndjeka N, Schnippel K, Master I, et al. High treatment success rate for multidrug-resistant and extensively drug-resistant tuberculosis using a bedaquiline-containing treatment regimen. Eur Respir J. 2018;52(6):1801528.

12. Ndjeka N, Ismail NA. Bedaquiline and clofazimine: successes and challenges. Lancet Microbe. 2020;1(4):e139-40.

13. World Health Organization. Technical manual for drug susceptibility testing of medicines used in the treatment of tuberculosis. Geneva: WHO; 2018.

14. World Health Organization. Meeting report of the WHO expert consultation on the definition of extensively drug-resistant tuberculosis, 27-29 October 2020. Geneva: WHO; 2021.

15. Wang L, Zhang H, Ruan Y, Chin DP, Xia Y, Cheng S, et al. Tuberculosis prevalence in China, 1990-2010; a longitudinal analysis of national survey data. Lancet. 2014;383(9934):2057-64.

16. Huo F, Luo J, Shi J, Zong Z, Jing W, Dong W, et al. A 10-year comparative analysis shows that increasing prevalence of Rifampin-resistant Mycobacterium tuberculosis in China is associated with the transmission of strains harboring compensatory mutations. Antimicrob Agents Chemother. 2018;62(4):e02303-e2317.

17. Mirzayev F, Viney K, Linh NN, et al. World Health Organization recommendations on the treatment of drug-resistant tuberculosis, 2020 update. Eur Respir J. 2021;57(6):2003300.

18. Gao M, Gao J, Xie L, Wu G, Chen W, Chen Y, et al. Early outcome and safety of bedaquiline-containing regimens for treatment of MDR- and XDR-TB in China: a multicentre study. Clin Microbiol Infect. 2021;27(4):597-602.

19. Liu Y, Gao M, Du J, Wang L, Gao J, Shu W, et al. Reduced susceptibility of Mycobacterium tuberculosis to bedaquiline during antituberculosis treatment and its correlation with clinical outcomes in China. Clin Infect Dis. 2020. https://doi.org/10.1093/cid/ciaa1002.
20. Huo F, Zhang F, Xue Y, Shang Y, Liang Q, Ma Y, et al. Increased prevalence of levofloxacin-resistant Mycobacterium tuberculosis in China is associated with specific mutations within the gyrA gene. Int J Infect Dis. 2020;92:241-6.

21. Zhang Z, Pang Y, Wang Y, Liu C, Zhao Y. Beijing genotype of Mycobacterium tuberculosis is significantly associated with linezolid resistance in multidrug-resistant and extensively drug-resistant tuberculosis in China. Int J Antimicrob Agents. 2014;43(3):231-5.

22. Cohen T, Jenkins HE, Lu C, McLaughlin M, Floyd K, Zignol M. On the spread and control of MDR-TB epidemics: an examination of trends in anti-tuberculosis drug resistance surveillance data. Drug Resist Updat. 2014;17(4-6):105-23.

23. Pang Y, Zhang Z, Wang Y, Wang S, Song Y, Zhao B, et al. Genotyping and prevalence of pyrazinamide- and moxifloxacin-resistant tuberculosis in China, 2000 to 2010. Antimicrob Agents Chemother. 2017;61(2):e02170-e2216.

24. Zhang QQ, Ying GG, Pan CG, Liu YS, Zhao JL. Comprehensive evaluation of antibiotics emission and fate in the river basins of China: source analysis, multimedia modeling, and linkage to bacterial resistance. Environ Sci Technol. 2015;49(11):6772-82

25. Nguyen TVA, Anthony RM, Banuls AL, Nguyen TVA, Vu DH, Alffenaar JC. Bedaquiline resistance: its emergence, mechanism, and prevention. Clin Infect Dis. 2018;66(10):1625-30.

26. Hartkoorn RC, Uplekar S, Cole ST. Cross-resistance between clofazimine and bedaquiline through upregulation of MmpL5 in Mycobacterium tuberculosis. Antimicrob Agents Chemother. 2014;58(5):2979-81.

27. Almeida Da Silva PE, Palomino JC. Molecular basis and mechanisms of drug resistance in Mycobacterium tuberculosis: classical and new drugs. J Antimicrob Chemother. 2011;66(7):1417-30.

28. Velayati AA, Farnia P, Ibrahim TA, Haroun RZ, Kuan HO, Ghanavi J, et al. Differences in cell wall thickness between resistant and nonresistant strains of Mycobacterium tuberculosis: using transmission electron microscopy. Chemotherapy. 2009;55(5):303-7.

\section{Publisher's Note}

Springer Nature remains neutral with regard to jurisdictional claims in published maps and institutional affiliations.
Ready to submit your research? Choose BMC and benefit from:

- fast, convenient online submission

- thorough peer review by experienced researchers in your field

- rapid publication on acceptance

- support for research data, including large and complex data types

- gold Open Access which fosters wider collaboration and increased citations

- maximum visibility for your research: over $100 \mathrm{M}$ website views per year

At BMC, research is always in progress.

Learn more biomedcentral.com/submissions 